\title{
REPENSAR EL SABER Y EL SABER HACER EDUCATIVO. UN ANÁLISIS CRÍTICO DESDE LAS PEDAGOGÍAS FEMINISTAS
}

\section{RETHINKING THE EDUCATIONAL KNOWLEDGE AND KNOW-HOW. A CRITICAL ANALYSIS FROM FEMINIST PEDAGOGIES}

\author{
Nicole Salas Salazar \\ Trabajadora Social. \\ Magíster (c) en Educación, mención en Currículum y Comunidad Educativa \\ Universidad de Chile \\ Santiago, Chile \\ salass.nicolef@gmail.com
}

\begin{abstract}
Resumen: A la luz de los tiempos que corren, se ha demostrado la necesidad de avanzar hacia la despatriarcalización de la educación. Esto, debido a que los espacios educativos en los cuales participamos están lejos de constituirse como instancias neutrales, toda vez que producen y reproducen múltiples sesgos existentes en un escenario global de legitimación de un conocimiento científico hegemónico de características androcéntricas, patriarcales, heteronormativas y etnocéntricas/colonialistas. Bajo dicho contexto, las pedagogías feministas proponen una relectura no sólo de la práctica pedagógica, sino que de igual manera de la reflexión del pensamiento epistemológico educativo. A partir de lo anterior, en esta
\end{abstract} oportunidad se desarrollarán sus principales postulados al respecto.

Palabras claves: Pedagogías feministas, género, educación, saber docente, práctica docente.

Abstract: In light of our current times, the need to advance towards the de-patriarchalization of education has been demonstrated. This is due to the fact that the educational spaces in which we participate are far from becoming neutral instances, since they produce and reproduce multiple biases that exist in a stage of global legitimation of a hegemonic scientific knowledge with andocentric, patriarchal, heteronormative and ethnocentric/colonist characteristics. In this context, feminist pedagogy proposes a rereading, not only of the pedagogical practice, but in the same way of the reflections on the educational epistemological thought. From the above, on this occasion the main postulates on the matter will be developed.

Key words: feminist pedagogies, gender, education, pedagogical knowledge, pedagogical practice 


\section{INTRODUCCIÓN}

El presente artículo busca proponer un análisis crítico, en clave feminista, a la tradición en la cual se han basado los supuestos epistemológicos y prácticos de la educación y de la pedagogía como disciplina.

En virtud de lo anterior, vale la pena destacar que, a lo largo de los años, tanto el movimiento social como las teorías feministas han aportado antecedentes relevantes para develar cómo el sistema educativo se constituye como un espacio reproductor, al interior de las mismas instituciones escolares, de las desigualdades de género que se observan fuera de ellas, impulsando la necesidad de concebir al currículum como un texto de género.

En nuestro contexto más inmediato, es posible recordar las diversas y multitudinarias movilizaciones estudiantiles feministas en Chile del Ilamado "mayo feminista", momento histórico en el que se interpeló fuertemente, tanto a las autoridades políticas como a las educacionales, por la cultura patriarcal presente a lo largo de todo el sistema educativo, razón por la cual se exigió con fuerza la demanda transversal por una educación no sexista y feminista. En este sentido, Palestro (2016, p.16) indica que "el sexismo en la educación es un factor clave en la reproducción de desigualdades y en la persistencia de distintas manifestaciones de violencia que impactan el vivir de las mujeres".

Por ello, el analizar los territorios educativos desde la óptica feminista está lejos de ser algo trivial, pues afecta directamente la vida de las personas y la construcción de sociedad. Ya que, "la educación es, a la vez, un asunto societal, político y técnico, que tiene su fundamento último y su legitimidad en los imaginarios de sociedad que se aspira a forjar en ese proceso" (Opertti, 2018, p. 43). De este modo, sabemos que estos imaginarios sociales presentes en la estructura macrosocial contribuyen a que se conciba el quehacer profesional desde una perspectiva universalista y hegemónica de carácter patriarcal, colonial y capitalista, y que a simple vista pueden resultar invisibles y naturalizados. En palabras de Boaventura de Sousa Santos, esta triada se expresa en la realidad de manera omnipresente pues, "para dominar efectivamente, tienen que ser imprudentes, feroces e incapaces de ser dominados" (Sousa Santos, 2020, p.35).

En este sentido, la educación en su dimensión hegemónica tiende a no generar instancias reales para reflexionar críticamente en torno al proceso pedagógico y a sus consecuencias, toda vez que el énfasis está puesto mayormente en el cumplimiento de objetivos predefinidos y en un currículo prescrito, los que se circunscriben, a su vez, al modelo económico capitalista. Es decir, prima una visión tecnócrata y muchas veces mercantilizada de la educación, donde la importancia radica únicamente en el hacer, más que en el pensar sobre dicho hacer o en el hacer para transformar. Frente a esto, Ossandón y Pinto (2018, p. 174) plantean la necesidad de abordar la organización del currículum como "un cambio de las relaciones sociales de poder, en que la institucionalidad local genere condiciones para una reflexión sobre los fines y procedimientos, y no solo sobre esto último".

Por otro lado, esta perspectiva tradicional reduce lo educativo solo a lo que ocurre al interior de los establecimientos educacionales, excluyendo los saberes circunscritos a otros 
espacios del conocimiento y los emanados de los grupos históricamente marginados, generando cierta jerarquización de saberes que van configurando y reproduciendo de manera sesgada la comprensión de la realidad. Así, el currículo prescrito "naturaliza relaciones de dominación/subordinación con base en jerarquías y roles sociales que guían la circulación de sus contenidos" (Hernández, 2019, p. 49), razón por la cual urge mantener viva la reflexión crítica que apunte a la transformación de la realidad desde una mirada feminista.

Tal como lo sostiene la educadora popular feminista Claudia Korol $(2007$, p. 16) "criticar una y otra vez las propias creencias parece ser el único camino para que nuestras ideas y nociones del mundo puedan ser vitales, fértiles, transformadoras". De esta manera, el presente texto pretende dar cuenta de diversos cuestionamientos $y$, a través de ellos, visibilizar las principales reflexiones teóricas que proponen las pedagogías feministas en este debate, a fin de extender la invitación a repensar las propias creencias y los mecanismos de producción de conocimiento y de ejercicio profesional, que se sitúan en un contexto hegemónico patriarcal/colonial/capitalista naturalizado.

En consecuencia, valdría la pena plantear las siguientes interrogantes al respecto: ¿Cuáles son los planteamientos que impulsan las pedagogías feministas? ¿Cuál es la relación entre feminismo y educación? ¿Cuál es la relación entre currículo prescrito y pedagogías feministas? ¿Es posible deconstruir o des-aprender un conocimiento construido desde una mirada hegemónica? ¿Cómo comenzar a gestar la despatriarcalización del pensamiento y de la práctica pedagógica?

Así, entendiendo la educación como un espacio en permanente tensión, "resulta del todo necesario identificar cuáles son los campos en disputa, cuáles son sus contradicciones y cuáles son las posibles hipótesis de trabajo a disponer sobre la mesa" (Nervi, 2007, p. 35). En razón de aquello, este ensayo espera aportar algunos elementos a esa discusión.

Siguiendo dicha idea, este texto parte de la base de que es necesario despatriarcalizar nuestra educación. En este sentido, se plantean las pedagogías feministas como herramientas teóricas y prácticas para propiciar espacios educativos reflexivos, cuestionadores y transformadores de la realidad. Pues, "la educación es un campo de lucha ideológico" (Abett de la Torre, 2014, p. 41), ergo, de constante disputa de saberes que van permeando constantemente el pensamiento y la acción.

Lo anterior, implica ciertos desafíos que nos permitirían analizar críticamente y generar cambios, tanto en la forma de producción de conocimiento educativo, como también en el quehacer pedagógico, entendiendo ambas dimensiones como un continuo perpetuador de la injusticia social. Y, a través de las siguientes líneas, se busca proponer algunas reflexiones que nos inviten a cuestionarnos, de manera individual y colectiva, un horizonte disciplinar que pueda estar en sintonía con las transformaciones sociales y políticas que nos sitúan contextualmente, pues la educación resulta ser un espacio de teorización, práctica y experiencia que no podemos dejar de mirar. 
En cuanto a la estructura del documento, las reflexiones se desarrollarán de la siguiente manera: En una primera instancia se identificarán algunas aproximaciones teóricas que nos ayudarán a comprender los principales postulados de las pedagogías feministas. Posteriormente, se profundizará sobre la crítica teórica que se levanta desde esta perspectiva, a la producción de conocimiento tradicional y al quehacer disciplinar pedagógico en los contextos educativos. Y, por último, se dará cuenta de algunas reflexiones finales que son posibles de plantear a modo de desafíos profesionales y epistemológicos.

\section{¿QUÉ ENTENDEMOS POR PEDAGOGÍA FEMINISTA?}

bell hooks (2017, p. 41), reflexionando sobre su propia historia como feminista, comenta que "la mayoría de nosotras habíamos sido socializadas por nuestros padres, y madres y por la sociedad para aceptar el pensamiento sexista". A partir de aquella experiencia -que bajo este paradigma ocupa un lugar central-, podemos inferir cómo no solo el sexismo, sino que también los roles de género, el racismo, el clasismo y el odio hacia las disidencias (entre otras exclusiones) están altamente naturalizadas por las sociedades en las que vivimos, siendo el sistema educativo uno de sus principales perpetuadores. En esta línea, es posible agregar que "la política educativa no puede ser comprendida de manera aislada, descontextualizada del marco sociohistórico concreto en el que cobra auténtico significado" (Torres, 2005, p. 13), por ello, la relevancia de propiciar la reflexión disciplinar de manera permanente se torna vital.

Bajo dicho contexto, es posible señalar que las pedagogías feministas están influenciadas por una relectura de los planteamientos de las teorías críticas en educación, las cuales introdujeron el análisis, principalmente a través de McLaren, "acerca de cómo los fenómenos de raza y sexo, además del de pertenencia de clase, impactan en la trayectoria escolar de los estudiantes" (Hirsch y Rio, 2015, p.80), esto no solo durante la escolaridad, sino que también a lo largo de todo el ciclo vital.

Así, "los debates pedagógicos feministas se han desarrollado en diálogo con las pedagogías críticas, coincidiendo ambas en pensarse como pedagogías liberadoras y revolucionarias" (Troncoso, Follegati y Stutzin, 2019, p. 6). Por tanto, éstas plantean aspectos asociados a cuestionar políticamente los mecanismos de subordinación presentes en los contextos educativos y de producción de conocimiento, pero desde un prisma transformador de lo existente, o en palabras de Giroux, respecto al rol del intelectual transformador, se debe “conseguir que lo pedagógico sea más político y lo político más pedagógico" (Hirsch y Rio, 2015, p.79), es decir, despojarse de la aparente neutralidad del ejercicio docente y asumir una posición de visibilización y de cuestionamiento a las relaciones de poder que caracterizan los contextos educativos.

En esta misma línea argumentativa, cabe señalar que "el perfil docente es parte sustancial de la respuesta a qué tipo de educación se requiere, para qué persona, ciudadanía y sociedad" (Opertti, 2018, p. 65). Lo anterior, en el entendido de que la reflexión y la práctica, de manera conjunta, permitirían avanzar hacia un horizonte de emancipación, lo que, a su vez, permitiría comenzar a vislumbrar uno de los principales puntos de encuentro entre educación y feminismo. 
En sintonía con aquello, volvemos a bell hook, quien al pensar en las aulas feministas sostiene que en dicho contexto se invita también "a reflexionar seriamente sobre la pedagogía en relación con la práctica de la libertad” (hooks, 1994 en Sánchez-Pardo, 2019, p. 112). Como vemos, esta concepción que, al estar inspirada en la pedagogía crítica de Freire, se caracteriza por una visión emancipadora, sustentada en la legitimación de la otra persona, en el diálogo y en la negociación, sin olvidar que estos procesos y experiencias son situadas.

Esto último nos lleva a problematizar también, que el conocimiento hegemónico que se enseña como "objetivo, verdadero y universal" no es tal, sino que es políticamente intencionado, ya que "la ciencia, [es] un texto discutible y un campo de poder", en palabras de Donna Haraway (1995, p. 317). En este sentido, la propuesta de entender los conocimientos como situados nos invita a adentrarnos al desafío de comprender que el proceso de enseñanza-aprendizaje debe contribuir a develar explícitamente que los contenidos trabajados son tales a partir de determinados contextos y formulados por un grupo específico de personas, las cuales, a su vez, ocupan lugares determinados en la estructura social, los que la mayoría del tiempo responde a posiciones de privilegio.

Entonces, "las pedagogías feministas toman como punto de partida la visibilización de las estructuras patriarcales que influyen en todas las dimensiones del desarrollo de las personas" (Martínez, 2018, p. 353), pues, como hemos mencionado, las diferencias que se sostienen durante la trayectoria educativa se traducen en desigualdades e injusticias sociales en la vida adulta, generando exclusión, discriminación, subordinación y precarización, no solo desde un análisis de clase, sino que de igual manera desde una perspectiva de género, raza, orientación sexual, etc.

De este modo, para una mayor contextualización, resulta valioso destacar que el desarrollo de las pedagogías feministas en el tiempo ha ido complejizándose cada vez más, enriqueciendo no solo su reflexión intelectual, sino que también su práctica en lo cotidiano. Un ejemplo de aquello, son las cinco fases o momentos de reestrucuturación curricular que Eliana Ortega (2018, p. 97) trae a discusión, a partir del aporte de otras pensadoras feministas.

Estas fases, que se plantean a continuación, no deben ser comprendidas, a juicio de la autora, como un camino lineal, toda vez que ellas van a estar supeditadas a las perspectivas, experiencias y voluntad de cambio de quienes integran el mundo académico y la gestión educativa: Fase 1) reconocer la existencia de las mujeres dentro de la historia oficial y hegemónica; Fase 2) concebir a las mujeres en la historia desde un rol excepcional y no desde su condición de marginalidad; Fase 3) diálogo entre el género, la clase, la raza y las sexualidades, como categorías de análisis fundamentales para la producción de conocimiento; Fase 4) cuestionamiento al rol jerárquico entre docente y aprendiz; Fase 5) comprender la transformación curricular a partir de las diferencias y la diversidad, y no más desde la homogeneidad y la generalización.

Para efectos de este artículo, se intentará plantear algunas ideas y reflexiones que recogen elementos contenidos en las últimas tres fases mencionadas, pues se tornan aportes valiosos y 
pertinentes a los desafíos contemporáneos que debe asumir el pensamiento y la práctica pedagógica.

\section{REPENSAR EL SABER Y EL SABER HACER EDUCATIVO DESDE LAS PEDAGOGÍAS FEMINISTAS}

Respecto a la construcción tradicional del conocimiento científico social, es posible señalar que "según Walsh, en las ciencias sociales y culturales en América Latina impera un modelo de ciencia que reproduce una serie de supuestos, sobre los que se establece toda una jerarquía académica" (Restrepo y Rojas, 2010, p. 142).

Siguiendo con esa misma línea argumentativa, en la década de los ochenta en Chile, la socióloga feminista Julieta Kirkwood (1987, p.44) sostenía que:

"El paradigma científico "reconocido" (patriarcal) no admite conocimientos que puedan poner en cuestión el orden posible (ideado y explicado por él) y, menos aún admite aquellos conocimientos (forma y contenido) que se atreven a postular ordenamientos teóricos alternativos".

En base a lo precedente, resulta relevante cuestionar las relaciones de poder que están imbricadas y caracterizan la tradición de considerar legítimo sólo el conocimiento que emerge desde lo hegemónico, es decir, desde el método científico. Y en este sentido, es posible señalar que "un primer aspecto relevante a la hora de pensar una educación feminista es la interpelación de las formas dominantes de producción de saberes" (Troncoso, Follegati y Stutzin, 2019, p. 7).

Por ello, las pedagogías feministas reconocen la validez de saberes que hoy se encuentran situados al margen de lo hegemónico. A modo de ejemplo, podemos mencionar, entre otros, los trabajos de las mujeres en el entorno académico patriarcal, los aportes que se posicionan desde los contextos latinoamericanos (racializados y originarios), o las experiencias que emergen desde los movimientos sociales que, a través de su acción política, buscan impugnar paradigmas. A raíz de lo anterior, es posible aseverar que "una educación en clave feminista se funda en un saber/conocer que postula una mirada/postura crítica hacia lo que se ha llamado verdades universales" (Ortega, 2018, p. 98), verdades que hoy asumen como propia la triada entre patriarcado, capital y colonialidad.

Sumado a las aproximaciones descritas, resulta interesante dar cuenta de la visión que aporta Claudia Korol (2007, p. 17), desde la educación popular, sobre el carácter autorreflexivo de las pedagogías feministas, y que podemos además hacer conversar con las cinco fases curriculares feministas expuestas con anterioridad:

"En esta perspectiva, falible, posible de ser criticada y modificada una y otra vez, que no aspira a ser universal ni válida para todo tiempo y lugar, la propuesta de "pedagogía popular feminista" actúa no como un límite, sino como una apertura. Es una manera de nombrar una posición en la batalla cultural, que cuestiona al conjunto de las relaciones de poder" 
Bajo esta perspectiva, vale la pena destacar la propuesta que se plantea de reivindicar una reflexión permanentemente abierta respecto de la producción de conocimiento y del quehacer educativo y sus consecuencias, dado lo cambiante de los procesos sociales. Un análisis similar, podemos encontrar en la reflexión de Irene Martínez, quien sostiene que:

"Las pedagogías feministas son emancipadoras y críticas. Invitan a la construcción de conocimiento desde una concepción abierta y diversa del mismo modo donde no existe un saber mejor que otro, si no múltiples formas de conocer la realidad" (Martinez, 2018, p. 356).

Sin embargo, el intentar comprender lo educativo desde la perspectiva feminista, resulta inevitable que la crítica, hacia los modos de producción de conocimiento, no esté vinculada con la práctica profesional, pues la teoría y el quehacer pedagógico no tienen sentido si no es bajo una sinergia constante. Por tanto, "las discusiones epistemológicas feministas deben nutrir en su riqueza y diversidad la práctica pedagógica feminista" (Troncoso, Follegati y Stutzin, 2019, p. 8), ya que, tal como se mencionó previamente, desde una perspectiva emancipadora, la reflexión es siempre pertinente en la medida que va acompañada de acciones concretas que puedan hacer carne dichos planteamientos en lo cotidiano.

Resulta de vital importancia entonces, comprender que el feminismo se funda también desde esa comprensión dialógica entre la teoría y la práxis, por ello, no es posible considerar una pedagogía, que se considere a sí misma como crítica, si deja fuera de sus planteamientos y supuestos epistemológicos la lectura feminista y, por consiguiente, que no propicie acciones de cambio. Por esta razón, podríamos circunscribir otro de los principales puntos de encuentro entre feminismo y pedagogía de la siguiente manera:

"la especificidad del área docente es apuntar, por un lado, a evidenciar que el conocimiento es poder y que por ser de origen masculino es sesgado, es "poder masculino" y, por el otro, que es posible construir un "contra - conocimiento" feminista que disuelva los privilegios sexistas" (Kirkwood, 1987, p. 141).

Asimismo, resulta muy relevante mencionar la instauración del debate en torno a la colonialidad como otra característica presente dentro de este saber hegemónico. Pues, "la idea de que el conocimiento era también un instrumento de colonización y que, por lo tanto, la descolonización implicaba la descolonización del saber y del ser (esto es subjetividad)" (Mignolo, 2010 , p. 9). Lo anterior cobra relevancia en este texto, pues como se mencionó inicialmente, las pedagogías feministas al concebirse como anti hegemónicas también asumen una postura crítica respecto a la dominación colonial, ya que, "al universalizar el conocimiento de la Modernidad, el currículum ejercita una violencia epistémica caracterizada por usar conceptos de un contexto cultural para interpretar otros contextos" (Hernández, 2019, p. 47). Recordemos nuevamente la importancia de la situacionalidad de Haraway.

En razón de aquello, valdría la pena problematizar en clave de género, las nociones de colonialidad del saber y colonialidad del poder, toda vez que "la colonialidad del saber supondría una especie de arrogancia epistémica” (Restrepo y Rojas, 2010, p. 137), generando por tanto, 
dada su característica patriarcal y androcéntrica, mecanismos de jerarquización y subordinación entre los mismos saberes, es decir, entre los "masculinos" y los "feminizados"; entre los "blancos" y los "racializados"; entre lo "heterosexual" y lo "disidente"; entre el "norte" y el "sur"; entre el "centro" y la "periferia", etc. En este sentido, Berger y Luckmann (2003, p. 47) nos plantean que "el conocimiento aparece distribuido socialmente, vale decir que diferentes individuos y tipos de individuos lo poseen en grados diferentes". Entonces, ¿en quienes ha estado encapsulado el conocimiento científico y educativo a lo largo de la historia? ¿a qué fines económicos ha tributado? ¿cuál es el rol de la educación ante este hecho? ¿es posible asumir una posición de indiferencia o de neutralidad desde los espacios educativos?

Aquí, la noción de interseccionalidad ${ }^{1}$ se torna un valioso aporte a la reflexión teórica, toda vez que la subordinación y opresión que generan los sistemas educativos capitalistas/patriarcales/coloniales, no afectan de manera homogénea ni en la misma intensidad al estudiantado. Así, "sin este enfoque interseccional no es posible entender cómo el patriarcado en la sociedad capitalista en que vivimos perpetúa la injusticia, la homofobia, la discriminación social y racial por la clase dominante"' (Ortega, 2018, p. 99).

Por ello, tal como lo plantea Iris Hernández (2019), uno de los principales desafíos para las pedagogías feministas, en el contexto de la hegemonía del currículum prescrito, radicaría en no caer en la reproducción de colonialismo, a lo que podríamos sumar también los otros modos de dominación (patriarcado y capitalismo). Y, recogiendo esta provocación, valdría la pena afirmar, que resulta difícil, por no decir imposible, pensar a las pedagogías feministas dentro de los márgenes y principios inmovilizadores que nos impone el legado curricular patriarcal de Tyler.

Sumado a lo anterior, y a propósito de lo subjetivo y su vínculo con el quehacer pedagógico, es importante aseverar que la disputa feminista en el campo educativo no es solo de contenidos o estructura, sino que de igual forma de sentidos comunes. Pues, adentrándonos al concepto de cultura propuesto por Geertz (2003, p.23), la escuela es la instancia por excelencia donde se reproduce cultura, ya que sostiene su funcionamiento a partir de los significados producidos por un proceso de interacción social constante entre los actores educativos.

En consecuencia, resulta clave analizar la incidencia de las prácticas pedagógicas en la construcción de ese sentido común capitalista, colonial y patriarcal, ya que estas provienen "de un sentido común inculcado en los seres humanos por la educación y el adoctrinamiento permanentes" (Sousa Santos, 2020, p. 35). Algunas expresiones de aquello son los contenidos implícitos que se enseñan en las escuelas; las didácticas que se utilizan para promover estos aprendizajes; el uso diferenciador del uniforme; las interacciones que se desarrollan dentro del espacio escolar; el ejercicio docente en dicho contexto; su sentido epistemológico y, las

\footnotetext{
${ }^{1}$ Concepto propuesto por Kimberly Crenshaw (1995) quien evidencia el cruce de opresiones que existe entre raza y género, visibilizando cómo se van configurando distintas estructuras de poder en base a ellas. Ver: "Mapping the Margins: Interseccionality, Identity Politics and violence. Againts Women of Color".

${ }^{2}$ Sin embargo, cabe señalar que la interseccionalidad, al calor del debate feminista, es un concepto que se ha ido complejizando en sus planteamientos y en su postura crítica ante la dominación. Ver: María Lugones (2005). "Multiculturalismo radical y feminismos de mujeres de color".
} 
consecuencias de todas estas dimensiones en la vida de las personas, sobre todo en la de los sectores históricamente excluidos.

Así, uno de los principales retos se centra en la invitación a ser conscientes de los significados que perpetuamos cotidianamente en los espacios escolares, en el entendido de que a través de dicho ejercicio podremos adherir o cuestionar esos elementos. Pues, tal como lo sostiene Blumer $(1992$, p.8) "la vida de un grupo humano es un proceso a través del cual los objetos van creándose, afirmándose, transformándose y desechándose". Por ello, las pedagogías feministas se plantean a sí mismas como transformadoras de la realidad y en permanente autorreflexión, ya que la neutralidad que se problematizaba anteriormente solo contribuye al mantenimiento del sistema de opresiones.

De este modo, podríamos vincular lo precedente con la fuerte influencia del currículum oculto que se da en la escuela, pues para Giroux y McLaren el estudio del currículum oculto resulta determinante "ya que en él se escencializan supuestos políticos antidemocráticos" (Hirsch y Rio, 2015, p.81), y al tratarse de aprendizajes implícitos, estos inciden fuertemente en las concepciones que durante la niñez se van definiendo respecto de los roles de género, y que se continúan legitimando en el continuo de la vida, imaginarios que, a su vez, también están cimentados bajo los supuestos hegemónicos que ya se han reiterado.

En cuanto a los contenidos explícitos que se enseña a diario en los centros educativos, resulta de vital importancia recordar que "mediante el currículum y los planes y programas, el sistema educativo selecciona los saberes que se espera que las nuevas generaciones aprendan" (Abett de la Torre, 2014, p. 41), por ello, el currículum tal y como lo conocemos, implica una selección de contenidos que nunca es neutral, pero que siempre es arbitraria y deliberada en este contexto de disputa constante.

Otro ejemplo es el lenguaje androcéntrico, pues tal como lo sostienen Berger y Luckmann (2003, p. 37) en su análisis sobre el conocimiento subjetivo "el lenguaje usado en la vida cotidiana me proporciona continuamente las objetivaciones indispensables y dispone el orden dentro del cual éstas adquieren sentido y dentro del cual la vida cotidiana tiene significado para mí". Así, se podría aseverar que "el feminismo es también una cuestión de lenguaje, pretendemos aquí entonces- la tarea de desmantelar el carácter androcéntrico, sexista y heteronormativo de lo que llamamos el lenguaje del amo" (Alonso et al, 2007, p. 107).

\section{REFLEXIONES FINALES}

A modo de conclusión, es posible señalar que, si bien se ha visibilizado la indudable fuerza del carácter patriarcal de la educación, vale la pena asumir esta realidad como una oportunidad disciplinar, en la medida de que esta situación puede promover la capacidad de agencia que poseen los y las docentes dentro de las aulas y, de este modo, situarse en un rol activo y crítico en la gestión curricular, más que en meros reproductores de lo hegemónico, siendo la formación docente una pieza crucial en este desafío. 
En este sentido, bell hooks plantea que "aprender es una revolución ... [un acto contrahegemónico]" (hooks, 1994 en Sánchez-Pardo, 2019, p. 112). Esta frase inspira a concebir la educación como un acto político y de permanente reflexión, tensión y disputa. Una revolución educativa, que tiene un carácter de resistencia a través de la reformulación de las prácticas pedagógicas y de la reconceptualización del pensamiento teórico, el cual debe asumir el desafío de deconstruir la reflexión epistemológica tradicional de la pedagogía y de las teorías curriculares y educativas, para abrir espacio a nuevos horizontes posibles.

Por tanto, urge transversalizar el pensamiento feminista en todas las dimensiones de la educación, vale decir, en la academia, en las universidades, en la formación docente, en el currículum, en las prácticas pedagógicas, en las formas de funcionamiento de los centros educativos, en los estilos de liderazgo, en los debates epistemológicos educativos, en la ciencias, etc, para que de este modo, las futuras generaciones puedan desarrollar procesos de enseñanzaaprendizaje basados en principios de justicia social y de rechazo a todos los mecanismos que generan opresión, y evitar así este doble ejercicio de aprender lo hegemónico para luego tener que des-aprenderlo.

Como vemos, se torna cada vez más necesario replantearnos los modos de pensamiento y las formas de vida, ya que "las propuestas que se hacen desde la pedagogía feminista consiste en tener una actitud constante de revisión de los referentes" (Martinez, 2018, p. 361), y es este entonces, una de las tareas cruciales, la cual consiste en tener la apertura de mirar desde otra óptica la realidad que nos rodea y, a través de ello, no solo cuestionar, sino que, sobre todo, transformar lo que se nos ha impuesto como realidad única y verdadera.

Así, recogiendo una visión del currículum como una práctica socio-constructiva, es posible sostener que tenemos la premura, cada vez más necesaria, de aventurarnos a:

"descubrir, en nuestros discursos y acciones organizadoras del currículum, las intenciones filosóficas, políticas, culturales e ideológicas que nos animan cuando nos posicionamos y decidimos hacer educación para la democracia, la justicia y la calidad de vida" (Ossandón y Pinto, 2018, p. 175).

En concordancia con lo anterior, y pensando en nuestros respectivos lugares de incidencia, Judith Butler (2015 en Caetano y de Garay, 2016, p. 266) sostiene que "el aula es el lugar donde los valores se construyen y así también las posibilidades de pensar de otra manera", en ese sentido, la invitación a transitar este camino desde la vereda de las pedagogías feministas está hecha, hagamos ahora que ocurra.

\section{BIBLIOGRAFÍA}

Abett de la Torre, P. (2016). Educación y Género: una reflexión sobre los principios de igualdad y diversidad en la educación chilena en el escenario de la reforma educativa actual. Paulo Freire. Revista De Pedagogía Crítica, (16), 35 - 47. 
Alonso, G., Herczeng, G, Lorenzi, B. y Zurbriggen, R. (2007). Espacios escolares y relaciones de género. En Korol, C. (Ed.). Hacia una pedagogía feminista. Géneros y educación popular (pp.107 - 128). Buenos Aires: Editorial El Colectivo y América Libre.

Berger, P. y Luckman, T. (1968) La construcción social de la realidad. Buenos Aires: Amorrortu.

Blumer, H. y Mugny, G. (1992) La psicología social. Modelos de interacción. Buenos Aires: CEAL.

Caetano, M. y de Garay, J. (2016). "Heteronormatividad y androcentrismo: ensayo sobre sus acciones curriculares". En Blazquez N., Castañeda M. (Ed). Lecturas críticas en investigación feminista (pp. 253-278). Cidade do México: UNAM.

Geertz, C. (2003). La interpretación de las culturas. Barcelona: Editorial Gedisa.

Haraway, D. (1995). Ciencia, cyborgs y mujeres. La reinvención de la naturaleza. Madrid: Ediciones Cátedra. Recuperado de: https://www.academia.edu/30099457/Donna_Haraway__Ciencia_cyborgs_y_mujeres

Hernández, I. (2019). Hacia un currículum feminista decolonial. Revista Nomadías. Dic. 28, 43-63.

Hirsch, D. y Rio, V. (2015). Teorías de la reproducción y teorías de la resistencia: una revisión del debate pedagógico desde la perspectiva materialista. Foro de Educación, 13 (18), pp. 6991.

hooks, b. (2017). El feminismo es para todo el mundo. Madrid: Traficantes de sueños

Kirkwood, J. (1987). Feminarios. Santiago de Chile: Ediciones Documentas

Korol, C. (2007). La educación como práctica de la libertad. Nuevas lecturas posibles. En Korol, C. (Ed.). Hacia una pedagogía feminista. Géneros y educación popular (pp. 9 - 22). Buenos Aires: Editorial El Colectivo y América Libre.

Martínez, I. (2018). Pedagogías Feministas: Estrategias una educación emancipadora y decolonial. Momento: diálogos em educação, 28 (3), pp. 350-365. Recuperado de: https://periodicos.furg.br/momento/article/view/8687/0

Mignolo, W. (2010) Desobediencia epistémica. Retórica de la modernidad, lógica de la colonialidad y gramática de la descolonialidad. Buenos Aires: Ediciones del Signo.

Nervi, M. (2007). ¿De qué hablamos cuando hablamos de pedagogía? En Nervi, M. y Nervi, H. (Eds.). ¿Existe la pedagogía? Hacia la construcción del saber pedagógico (pp. 35 - 51). Santiago: Editorial Universitaria 
Opertti, R. (2018). 10 notas para apuntalar una agenda de Transformaciones educación 2030. En Arratia, A. y Ossandón, L. (Eds.). Políticas para el Desarrollo del Currículum: Reflexiones y Propuestas (pp. 39 - 82). Santiago: MINEDUC - UNESCO

Ortega, E. (2018). Señas para una Educación Feminista. Revista Nomadías. Jul. 25, 91-100.

Ossandón, L. y Pinto, R. (2018). El currículum nacional y la descentralización: políticas, institucionalidad y saberes. En Arratia, A. y Ossandón, L. (Eds.). Políticas para el Desarrollo del Currículum: Reflexiones y Propuestas (pp. 155 - 184). Santiago: MINEDUC - UNESCO

Palestro, S. (2016). Androcentrismo en los textos escolares. En Red Chilena contra la Violencia hacia las Mujeres, Educación No Sexista. Hacia una real transformación. Santiago: Andros Impresiones, 15-25.

Restrepo, E. y Rojas, A. (2010) Inflexión decolonial: fuentes, conceptos y cuestionamientos. Popayán, Colombia, Universidad del Cauca, Instituto Pensar, Universidad Javeriana.

Sánchez-Pardo, E. (2019). Es una gran responsabilidad ser uno mismo. Lo personal y lo político de nuevo a debate en la pedagogía feminista. En B. Revelles y A. González (Eds.), Género en la educación. Pedagogía y responsabilidad feministas en tiempos de crisis política (pp.101119). Madrid: Ediciones Morata.

Sousa Santos, B. (2020). La cruel pedagogía del virus. Buenos Aires: CLACSO

Torres, J. (2005). El currículum oculto. Madrid: Ediciones Morata.

Troncoso Perez, L., Follegati, L., y Stutzin, V. (2019). Más allá de una educación no sexista: aportes de pedagogías feministas interseccionales. Pensamiento Educativo. Revista de Investigación Educacional Latinoamericana, 56(1), 1-15. Recuperado de:

https://pensamientoeducativo.uc.cl/index.php/pel/article/view/1092 